\title{
The Influence of Adsorbates on the Growth Mode of Gold Islands Electrodeposited on the Basal Plane of Graphite
}

\author{
H. Martín, ${ }^{\dagger}$ P. Carro, ${ }^{\dagger}$ A. Hernández Creus, ${ }^{\dagger}$ S. González, ${ }^{\dagger}$ G. Andreasen, ${ }^{\ddagger}$ \\ R. C. Salvarezza, ${ }^{\ddagger}$ and A. J. Arvia*,‡ \\ Departamento de Química Física, Universidad de La Laguna, La Laguna, Tenerife, Spain, \\ and Instituto de Investigaciones Fisicoquímicas Teóricas y Aplicadas (INIFTA) (UNLP, \\ CONICET, CIC BsAs), Sucursal 4, Casilla de Correo 16, (1900) La Plata, Argentina
}

Received August 20, 1999. In Final Form: November 23, 1999

\begin{abstract}
Gold electrodeposition on $\mathrm{C}(0001)$ from aqueous $0.5 \mathrm{mM} \mathrm{AuCl}{ }_{3} \cdot \mathrm{HCl}$, in the range of apparent charge density $\left(6 \leq q \leq 10 \mathrm{mC} / \mathrm{cm}^{2}\right)$ and under mass transport kinetic control from the solution side, results in the formation of nanometer to micrometer size $\mathrm{Au}(111)$ islands. These islands consist of a small threedimensional (3D) central core and large quasi-two-dimensional (2D) dendritic branches. Ex situ scanning tunneling microscopy (STM) imaging data show that the shape of islands is determined by anisotropic surface diffusion contributions. The addition of citric acid to the plating solution hinders branching and promotes 3D island growth. The adsorption of citric acid at step edges hinders interterrace gold atom surface diffusion, as concluded from in situ STM imaging. Conversely, the addition of an excess of sodium chloride enhances 2D island growth and dense branching formation. These results can be explained considering that the presence of adsorbates either increases or decreases the height of activation energy barriers for interterrace surface diffusion. In this way, the change of the island aspect ratio with the nature of the additive can be explained.
\end{abstract}

\section{Introduction}

The heteroepitaxial growth of a solid phase on a welldefined crystalline foreign substrate occurring far from conditions of equilibrium is a complex process involving the initial formation of nuclei, their growth forming either two-dimensional (2D) or three-dimensional (3D) crystals, and finally, the overlapping of these crystals covering most of the substrate surface. ${ }^{1}$ The first and second steps lead to a solid surface consisting of patches of the substrate and islands of the new solid phase. Under certain experimental conditions, these islands exhibit fractal patterns. ${ }^{2}$ The kinetics and mechanism of crystal growth at the molecular level and Laplacian fields built up around the growing crystal determine the characteristics of patterns at a certain stage of growth. This phenomenon has been proved for a number of patterns produced from different growth models. ${ }^{3}$

Different growth models have been proposed to describe the initial stages of island formation on a crystalline substrate, such as the deposition, diffusion, and aggregation (DDA) model. ${ }^{2}$ According to this model, particles deposited either on the substrate or on growing islands move by random walk to be incorporated into island edges by surface diffusion. Patterns resulting from this model consist of $2 \mathrm{D}$ islands, their shape resembling closely that expected from either diffusion-limited aggregation (DLA) ${ }^{3}$, dendrite-like, ${ }^{4}$ or dense radial aggregation models, ${ }^{2}$ de-

* Author to whom correspondence should be sent. Telephone: 54-221-425-7430/7291. Fax: 54-221-425-4642. E-mail:ajarvia@ inifta.unlp.edu.ar.

† Departmento de Química Física.

¥ Instituto de Investigaciones Fisicoquímicas/Teóricas y Aplicadas

(1) Budevski, E.; Staikov, G.; Lorenz, W. J. In Electrochemical Phase Formation and Growth, VCH: Weinheim, 1996.

(2) Barabasi, A. L.; Stanley, E. In Fractal Concepts in Surface Growth; Cambridge University Press: Cambridge, 1995.

(3) Meakin, P. In The Fractal Approach to the Heterogeneous Chemistry, Avnir, D., Ed.; Wiley: New York, 1989; p 131, and references therein. pending on subtle differences in the surface diffusion characteristics of deposited particles. The DDA model, although developed as a homoepitaxial 2D model, generates growth patterns closely comparable to those resulting from the heteroepitaxial growth of a new phase. ${ }^{4-6}$

Branched quasi-2D islands have been produced by vapor phase deposition on (111) metal faces where two distinct types of steps are present; namely, A-type (in the (100) direction) and B-type (in the (111) direction). ${ }^{4}$ For B-type steps, lower activation energy barrier for adatom surface diffusion than for A-type steps have been calculated. ${ }^{4}$ The formation of dendritic branching also requires anisotropic corner diffusion to stabilize the tip against tip splitting. ${ }^{4}$ Very recently it has been found that the shape of branched islands can be drastically changed by the presence of adsorbates at step edges, which produces a change in the height of energy barriers for surface atom diffusion. ${ }^{7}$

The electrodeposition of gold on $\mathrm{C}(0001)$ has been employed to grow quasi-2D branched islands. ${ }^{8}$ In this case, gold electrodeposition undergoes within a potential window $(\Delta E)$ that is negative with respect to the potential of zero charge $\left(E_{\mathrm{pzc}}\right)$ of $\mathrm{Au}(111)$ in the plating solution. The kinetics of the process is under mass-transport rate control. The origin of dendritic gold islands has been related to the existence of activation energy barriers for surface atom interterrace diffusion and anisotropic corner diffusion for gold atoms, ${ }^{4,5}$ the activation energy involved in the overall process being $11 \pm 3 \mathrm{kcal} / \mathrm{mol} .{ }^{9}$ From the knowledge of this system just by adequately changing the composition

(4) Brune, H.; Roder, H.; Bromann, K.; Kern, K.; Jacobson, J.; Stoltze, P.; Jacobson, K.; Norskov, J. Surf. Sci. 1996, 349, L115.

(5) Hwang, R. Q.; Schroder, J.; Gunther, G.; Behm, R. J. Phys. Rev. Lett. 1991, 67, 3279

(6) Mueller, B.; Nedelman, L.; Fischer, B.; Brune, H.; Barth, J.; Kern, K. Phys. Rev. Lett. 1998, 80, 2645.

(7) Kalff, M.; Comsa, G.; Michely, T. Phys. Rev. Lett. 1998, 6, 1255.

(8) Martín, H.; Carro, P.; Hernández Creus, A.; González, S : Salvarezza, R. C.; Arvia, A. J. Langmuir 1997, 13, 110.

(9) Martín, H.; Carro, P.; Hernández Creus, A.; González, S.; Salvarezza, R. C.; Arvia, A. J. J. Phys. Chem. B 1999, 103, 3900. 
of the plating solution, it is possible to investigate the influence of adsorbates on the growth mode of gold islands using a low additive concentration as commonly employed in metal electroplating. ${ }^{10}$

In this work, the influence of a small amount of either citric acid or sodium chloride added to the $\mathrm{AuCl}_{4}{ }^{-}$ioncontaining-plating solution on the morphology of gold islands is studied by scanning tunneling microscopy (STM). In contrast to the presence of sodium chloride, citric acid eliminates branching and promotes the growth of rounded quasi-3D islands. This effect of citric acid on the gold island growth mode is due to an increase in the height of activation energy barriers for gold atom surface diffusion at step edges that interfere with interterrace and corner surface diffusion. This conclusion is verified by following the decay of monatomic high gold islands on $\mathrm{Au}(111)$ observed by in situ sequential STM imaging in the absence of $\mathrm{AuCl}_{4}{ }^{-}$ions in the solution.

The change in the growth mode induced by the presence of different adsorbates can be explained either by a hindrance to the interterrace gold atom surface diffusion by molecular adsorption at step edges in the case of citric acid or by its enhancement in the presence of sodium chloride.

\section{Experimental Section}

The electrodeposition of gold on a freshly exfoliated C(0001) working electrode $\left(0.5 \mathrm{~cm}^{2}\right)$ was made at $298 \mathrm{~K}$ using a conventional three-electrode glass-made electrochemical cell. A polycrystalline platinum and a saturated calomel electrode (SCE) as counter electrode and reference electrode, respectively, were utilized. Potentials in the text are referred to the SCE scale.

For gold plating, aqueous $0.5 \mathrm{mM} \mathrm{AuCl}_{3} \cdot \mathrm{HCl}+0.5 \mathrm{M} \mathrm{NaClO}_{4}$ $+10^{-2} \mathrm{M} \mathrm{HClO}_{4}$ (Solution I) was used as such or with the addition of either citric acid $(C=0.1 \mathrm{mM})$ (Solution II) or sodium chloride $\left(C=0.92 \times 10^{-2} \mathrm{M}\right)$ (Solution III). Gold electrodeposition was made at constant potential $(E)$ in the range $-0.1 \leq E \leq 0 \mathrm{~V}$. The $(1 \times 1) \mathrm{Au}(111) \leftrightarrow \mathrm{Au}(22 \times \sqrt{3})$ surface reconstruction was used for monitoring the formation and disappearance of gold islands. For these experiments, aqueous $0.5 \mathrm{M} \mathrm{NaClO}_{4}+10^{-2} \mathrm{M} \mathrm{HClO}_{4}$ was used as such or with the addition of citric acid. Solutions were prepared from analytical reagent-grade chemicals and MilliQ*-Millipore water, and deaerated by bubbling purified argon for $2 \mathrm{~h}$ prior to gold electrodeposition.

The formation of gold islands was investigated in the range of apparent charge density $\left(6 \leq q \leq 10 \mathrm{mC} / \mathrm{cm}^{2}\right)$ by combining conventional electrochemical techniques and STM. Cathodic polarization curves covering the range $0.9 \geq E \geq-0.1 \mathrm{~V}$, at the potential scan rate $V=1 \times 10^{-3} \mathrm{~V} / \mathrm{s}$, were recorded with a PAR instrument consisting of EQ\&G 173, 175, 178, and 179 units. Potentiostatic current transients were recorded for $E<0.1 \mathrm{~V}$.

Gold island formation on C(0001) was followed by ex situ STM imaging. In this case, the working electrode was removed from the electrochemical cell, rinsed by successive immersions in water, and finally dried under argon at room temperature. Subsequently, STM images were obtained using a Nanoscope IIE instrument (Digital Instruments, Santa Barbara, CA) with Pt-Ir Nanotips operating in air. This operation mode was feasible because of the stability of the electrode surface in air. Images were taken at 0.5 $\mathrm{nA}$ tunneling current, $0.2 \mathrm{~V}$ bias voltage, and scan rates usually in the order of $0.5 \mathrm{~Hz}$ for images $3.5 \times 3.5 \mu \mathrm{m}^{2}$ in size. For these conditions, either damage to or removal from the substrate of gold islands by the tip could be prevented.

The potential dependent $(1 \times 1) \mathrm{Au}(111) \leftrightarrow \mathrm{Au}(22 \times \sqrt{3})$ surface reconstruction was followed by in situ STM imaging. For these runs, flame-air-treated $\mathrm{Au}(111)$ substrates (Robax glass AF Berliner Glass KG, Germany) working electrode, a Pd/ $\mathrm{H}_{2}$ reference electrode, and a large area gold counter electrode mounted in a Nanoscope II electrochemical cell were utilized.

10) Lowenheim, F. A. Modern Electroplating, Wiley: New York,
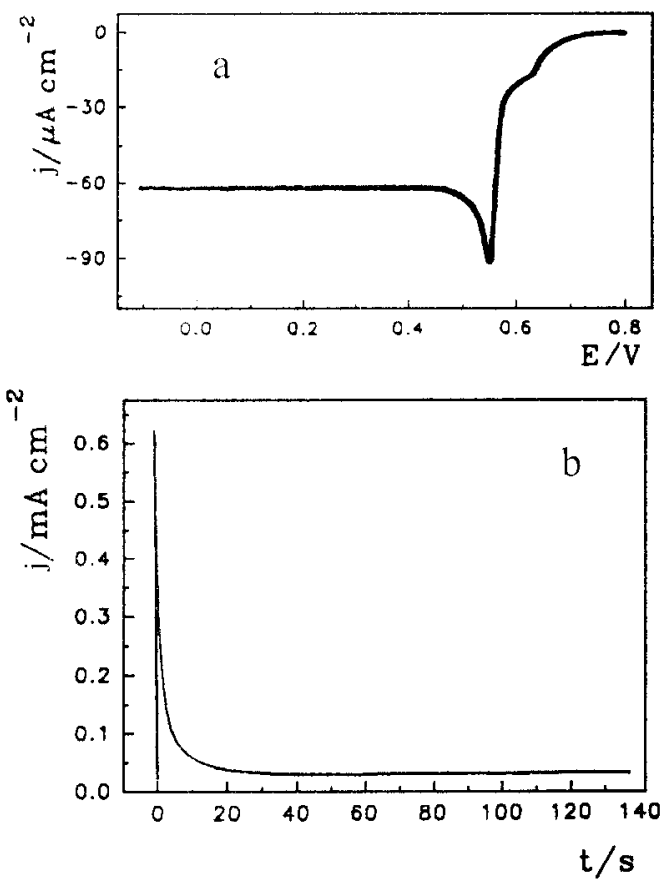

Figure 1. (a) Apparent current density vs potential plot for gold electrodeposition from aqueous $5 \times 10^{-4} \mathrm{M} \mathrm{AuCl}_{3} \cdot \mathrm{HCl}+$ $0.5 \mathrm{M} \mathrm{NaClO}_{4}+0.01 \mathrm{M} \mathrm{HClO}_{4}$ on $\mathrm{C}(0001)$ made at $0.001 \mathrm{~V} / \mathrm{s}$ and $298 \mathrm{~K}$. A clear cathodic current plateau for $E<0.4 \mathrm{~V}$ can be observed. (b) Typical potentiostatic current transient for $E$ $=-0.1 \mathrm{~V}$ run in the same solution.

\section{Results}

3.1. Electrochemical Data. The apparent current density $(j)$ vs applied potential (E) plots for gold electrodeposition on $\mathrm{C}(0001)$ at $V=0.001 \mathrm{~V} / \mathrm{s}$, started from $E$ $=0.8 \mathrm{~V}$ in the negative direction (Figure $1 \mathrm{a}$ ), show a first cathodic wave in the range $0.7-0.6 \mathrm{~V}$, a current peak at $E \cong 0.55 \mathrm{~V}$, and a cathodic current plateau $\left(j_{\mathrm{L}}\right)$ extending from $0.45 \mathrm{~V}$ downward to $-0.1 \mathrm{~V}$. The first cathodic wave with a half-wave potential close to $0.66 \mathrm{~V}$ is related to the first electroreduction stage of $\left[\mathrm{AuCl}_{4}\right]^{-}$ion according to

$$
\left[\mathrm{AuCl}_{4}\right]^{-}(\mathrm{aq})+2 \mathrm{e}^{-}=\left[\mathrm{AuCl}_{2}\right]^{-}(\mathrm{aq})+2 \mathrm{Cl}^{-}(\mathrm{aq})
$$

The second limiting current plateau with a half-wave potential at $\sim 0.55 \mathrm{~V}$ corresponds to gold electrodeposition from both $\left[\mathrm{AuCl}_{2}\right]^{-}(\mathrm{aq})$ and $\left[\mathrm{AuCl}_{4}\right]^{-}$(aq) species

$$
\begin{gathered}
{\left[\mathrm{AuCl}_{2}\right]^{-}(\mathrm{aq})+\mathrm{e}^{-}=\mathrm{Au}(\mathrm{s})+2 \mathrm{Cl}^{-}(\mathrm{aq})} \\
{\left[\mathrm{AuCl}_{4}\right]^{-}(\mathrm{aq})+3 \mathrm{e}^{-}=\mathrm{Au}(\mathrm{s})+4 \mathrm{Cl}^{-}(\mathrm{aq})}
\end{gathered}
$$

The standard equilibrium potential of reaction 3 in the working solution is $E^{\circ}=0.757 \mathrm{~V} .{ }^{11}$ The dependence of $j_{\mathrm{L}}$ on the $\mathrm{AuCl}_{4}{ }^{-}$ion concentration $(C)$ fits the equation for a mass-transport-controlled electrochemical process. ${ }^{8,9}$

Potentiostatic current transients (Figure 1b), recorded in the potential range $0.2 \geq E \geq-0.1 \mathrm{~V}$, also reflect the mass-transport control for gold electrodeposition because they yield linear $j$ vs $t^{-1 / 2}$ plots, as expected from SandCottrell's equation. ${ }^{12}$

In the solution composition range covered by this work, neither the addition of citric acid nor of sodium

(11) Schmid, G. M.; Curley-Fiorino, M. E. In Encyclopedia of Electrochemistry of the Elements; Bard, A. J., Ed.; Marcel Dekker: New York, 1975; Vol. IV, p 87.

(12) Scharifker, B.; Hills, G., Electrochim. Acta 1983, 28, 879. 


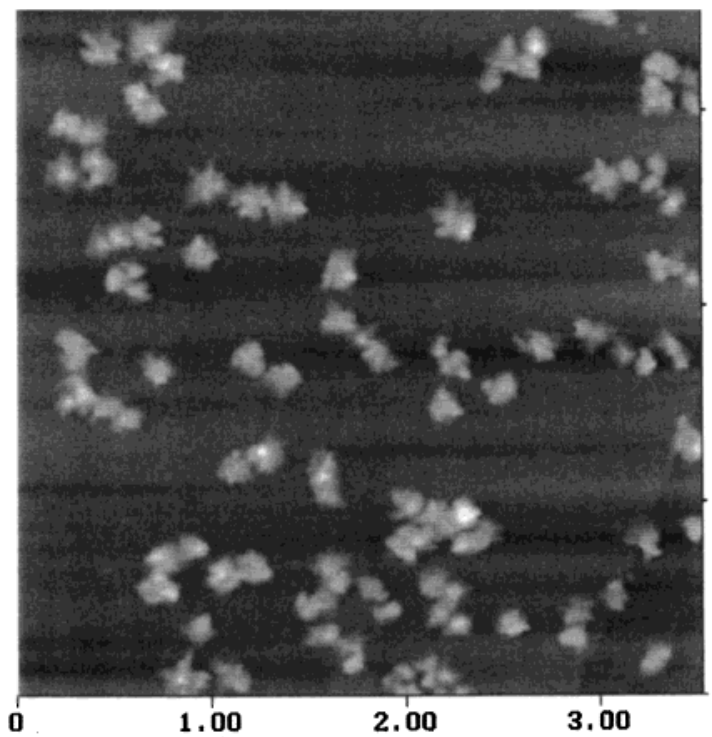

a

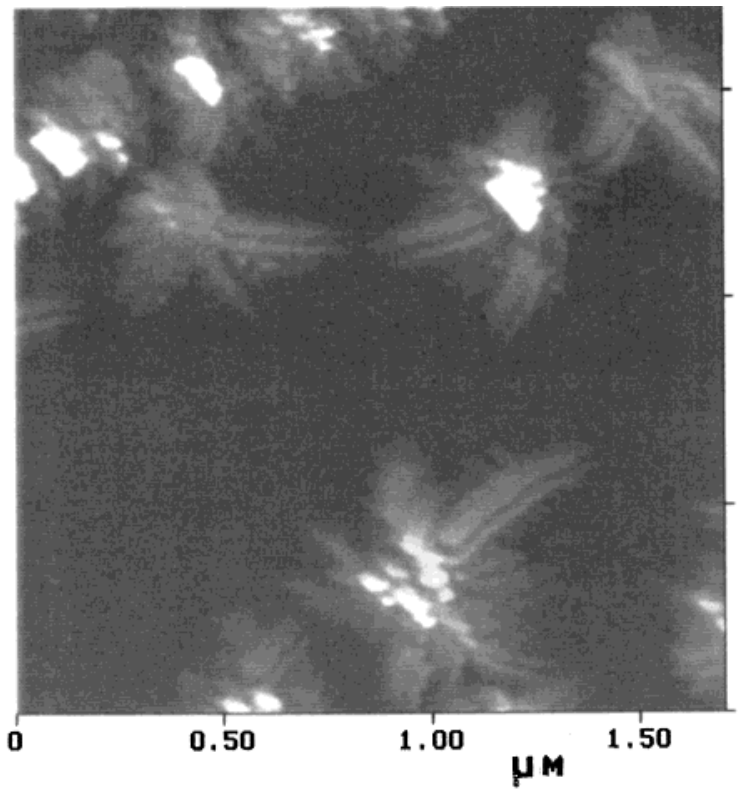

b

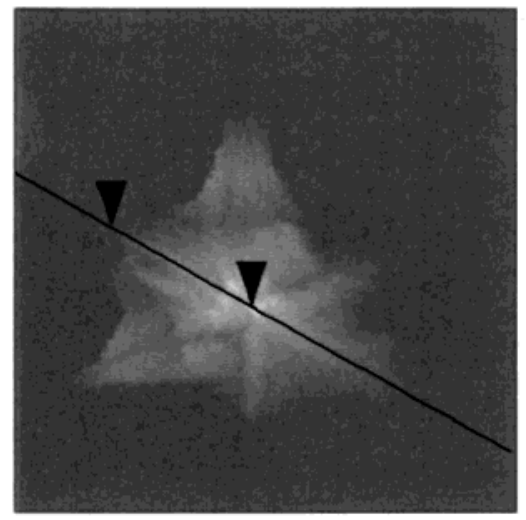

C

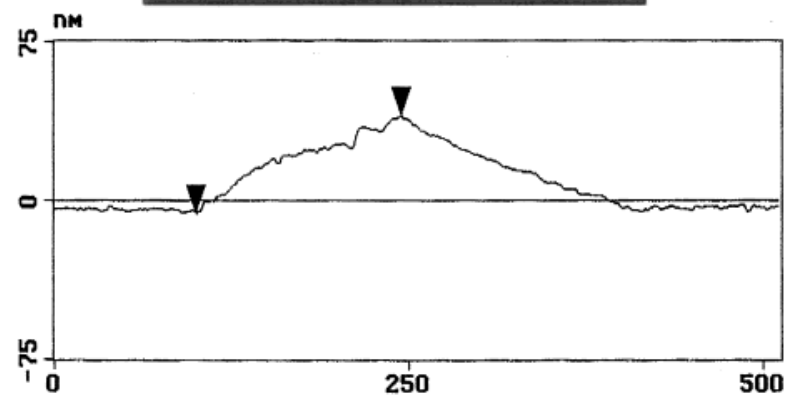

Figure 2. Ex situ STM images (topographic mode) of gold islands on $\mathrm{C}(0001)$ produced from aqueous $5 \times 10^{-4} \mathrm{M} \mathrm{AuCl}_{3} \cdot \mathrm{HCl}+$ $0.5 \mathrm{M} \mathrm{NaClO}_{4}+0.01 \mathrm{M} \mathrm{HClO}_{4}$ under constant flux $(E=-0.1 \mathrm{~V})$. (a) $3.5 \times 3.5 \mu \mathrm{m}^{2}$ image, $q=6 \mathrm{mC} / \mathrm{cm}^{2} ;(\mathrm{b}) 1.7 \times 1.7 \mu \mathrm{m}^{2}, q=$ $10 \mathrm{mC} / \mathrm{cm}^{2}$. (c) Cross section of a typical island grown for $q=6 \mathrm{mC} / \mathrm{cm}^{2}$.

chloride to the plating solution produces significant changes in the $j$ vs $E$ profile and the value of $j_{L}$. The same conclusion comes out from the analysis of the corresponding $j$ vs $t^{-1 / 2}$ plots. These results indicate that there is no influence of those species added to the solution on the mass transport rate control from the solution side.

3.2. STM Imaging. 3.2.1. Ex Situ STM Imaging of Gold Islands on C(0001). After gold electrodeposition on C(0001) from Solution I for $q=6 \mathrm{mC} / \mathrm{cm}^{2}$, ex situ STM images $\left(3.5 \times 3.5 \mu \mathrm{m}^{2}\right)$ (Figure $\left.2 \mathrm{a}\right)$ show that only a relatively small fraction of $\mathrm{C}(0001)$ is covered by gold islands. These islands are nucleated at random on C(0001) terraces. Each island (Figure $2 \mathrm{~b}$ ) consists of a large number of tiny gold crystals, with a high rounded central core and branches without tip splitting, as expected for dendrites, emerging at well-defined angles. In general, branches with the longest longitudinal cross section emerge at $120^{\circ}$ angles. The longitudinal cross section of a single branch (Figure 2c) reveals a sequence of terraces 25-30 $\mathrm{nm}$ in size decreasing in height from the core border toward the branch tip by one- or two-atom-high steps. ${ }^{9}$

A typical gold island can be described in terms of the maximum island radius $(R)$, the radius enclosing each gold crystal, and the maximum island height $\left(h_{\mathrm{M}}\right)$. Previous results from this system have shown that $R$ and $t$ obey the kinetic law $\langle r\rangle \propto t^{n}$ with $n=0.25 \pm 0.05$ (Figure 3)..$^{9}$ This relationship is consistent with a surface-diffusioncontrolled radial island growth. ${ }^{13}$ The aspect ratio, $f_{\mathrm{I}}=$ $h_{\mathrm{M}} / R$, derived from the cross section analysis of gold islands

(13) Schwoebel, R. J. J. Appl. Phys. 1969, 40, 614; Smilauer, P.; Vvendenski, D. D. Phys. Rev. B 1995, 52, 14263. 


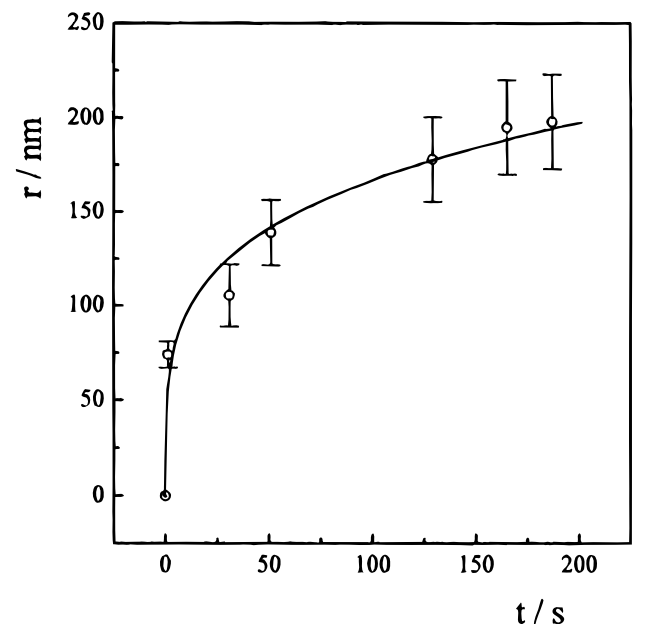

Figure 3. (a) Plots of $\langle r\rangle$ vs $t$ for the range $0.6 \leq q \leq 25 \mathrm{mC} / \mathrm{cm}^{2}$; $T=298 \mathrm{~K}$. The continuous trace corresponds to the $r \propto t^{1 / 4}$ relationship. Data taken from ref 9.

(Figure 2c) produced from Solution I, is $f_{\mathrm{I}}=0.30 \pm 0.05$, irrespective of $q$.

Gold electrodeposition on C(0001) from Solution II for $q=6 \mathrm{mC} / \mathrm{cm}^{2}$ (Figure 4 a) produces a remarkable increase in the surface density of gold islands and a drastic change in island morphology. In this case, branching is largely hindered and the development of rounded 3D islands is favored (Figure 4b). From the cross section analysis (Figure 4c) of gold islands resulting from Solution II (Figure 4a), $\mathrm{f}_{\mathrm{II}}=0.66 \pm 0.05$. This figure reveals a change in the growth mode of gold islands from a largely anisotropic to an almost isotropic one caused by the presence of citric acid. This drastic change in the growth mode suggests that gold atom surface diffusion on gold is considerably hindered by the presence of citric acid, leading to a decrease in the average size of islands, an increase in the surface density of gold islands, and a tendency to promote a quasi-3D growth.

Conversely, those islands that were grown in Solution III (Figure 5a,b) exhibit a large number of branches, an increase in the average island size, and correspondingly, a decrease in the density of islands, as compared with those found from Solutions I and II. The increase in the island size correlates with the increase in the size of branches leading to $f_{\text {III }}=0.14 \pm 0.03$ (Figure $5 \mathrm{~b}$ ). The decrease in the value of $f_{I I I}$ as compared with $f_{I I}$ means that the presence of an excess of chloride ions in the solution promotes 2D gold island growth.

3.2.2. In Situ STM Imaging of Gold Islands on Au(111). To demonstrate the influence of citric acid on the surface mobility of gold atoms on $\mathrm{Au}(111)$, a flame airtreated $\mathrm{Au}(111)$ electrode was held first at $E=0.20 \mathrm{~V}$ in aqueous $0.5 \mathrm{M} \mathrm{NaClO}_{4}+10^{-2} \mathrm{M} \mathrm{HClO}_{4}$ (i.e., a $\mathrm{AuCl}_{4}{ }^{-}$ ion-free working solution). As already reported for $E<$ $E_{\mathrm{pzc}}=0.25 \mathrm{~V}$, the zero charge potential of $\mathrm{Au}(111),{ }^{14}$ the $22 \times \sqrt{3}$ surface reconstruction of $\mathrm{Au}(111)$ is clearly observed in the STM images (Figure 6a). It is characterized by parallel pairs of stripes separated by the distance $\mathbf{a}=$ $6.6 \mathrm{~nm}$. When the value of $E$ is changed from 0.20 to 0.35 $\mathrm{V}\left(E>E_{\mathrm{pzc}}\right)$, the lifting of the $22 \times \sqrt{3}$ surface reconstruction takes place leading to $(1 \times 1) \mathrm{Au}(111)$, and simultaneously the excess of gold atoms remaining at the surface form atomically smooth one atom high gold islands (Figure 6b,c). When $E$ is changed backward from 0.35 to $0.20 \mathrm{~V}$, the reverse process can be observed; that is, $22 \times$

(14) Kolb, D. M.; Dakkouri, A. S.; Batina, N. In Nanoscale Probes of the Solid/Liquid Interface, Gewirth, A. A.; Siegenthaler, H., Eds.; Kluwer Academic Publishers: Dordrecht, 1995; p 263. $\sqrt{3}$ surface reconstruction rapidly reappears and gold islands decrease in size and finally disappear (Figure 6d). This process involves the displacement of gold atoms from island edges, their surface diffusion on the Au(111) terrace, and their incorporation into the $22 \times \sqrt{3}$ surface structure, a process that is known to occur under gold atom surface diffusion control. ${ }^{14}$

Similar experiments were made using aqueous $0.5 \mathrm{M}$ $\mathrm{NaClO}_{4}+10^{-2} \mathrm{M} \mathrm{HClO}_{4}+0.1 \mathrm{mM}$ citric acid starting from the $22 \times \sqrt{3}$ reconstructed surface $(E=0.20 \mathrm{~V})$ (Figure 7a). This image shows the parallel pairs of stripes already referred to, although in this case the value $\mathbf{a}=$ $8.0 \mathrm{~nm}$ is greater than that derived from Figure 6a. When the value of $E$ is changed from 0.20 to $0.35 \mathrm{~V}$ (Figure $7 \mathrm{~b}$ ), the simultaneous formation of the $(1 \times 1) \mathrm{Au}(111)$ surface structure and one atom high gold island takes place at a rate lower than that found in aqueous $0.5 \mathrm{M} \mathrm{NaClO}_{4}+$ $10^{-2} \mathrm{M} \mathrm{HClO}_{4}$. Subsequently, when $E$ is set back to 0.20 $\mathrm{V}$ (Figure 7c), the reappearance of the $22 \times \sqrt{3}$ reconstructed surface and the decrease in gold island average size occur at a rate lower than that observed in the absence of citric acid in the solution. Preliminary results showed that for islands $5 \mathrm{~nm}$ in average size, the island diameter decays almost linearly with time at the average rates of $0.026 \mathrm{~nm} / \mathrm{s}$ for Solution I and $0.013 \mathrm{~nm} / \mathrm{s}$ for Solution II. This is proof of the influence of citric acid on gold atom surface diffusion on $\mathrm{Au}(111)$.

Therefore, it can be concluded that the presence of citric acid slows down the surface diffusion of gold atoms moving from island edges to those surface sites involved in the 22 $\times \sqrt{3}$ surface reconstruction and vice versa. Furthermore, the fact that the influence of citric acid manifests itself at the level of one atom high islands can be taken as an indication that the citric molecule-gold atom interaction operates at terrace steps, probably involving a weak interaction there. The attempts to image citric acid molecules at the gold surface were probably unsuccessful because of either their own surface mobility or removal from the surface by the tip. However, the atomic lattice of $\mathrm{Au}(111)$ at terraces was easily obtained.

\section{Discussion}

4.1. Processes Involved in Gold Island Formation of C(0001). The comparison of electrochemical data and STM images for gold electrodeposition, irrespective of the additive, show that in the range $6<q<10 \mathrm{mC} / \mathrm{cm}^{2}$, the degree of surface coverage of C(0001) by gold islands is no greater than 0.3 . In fact, gold electrodeposition takes place at certain defective sites of $\mathrm{C}(0001)$ where the formation of nuclei is favored. ${ }^{8}$ Then, in the range of $q$ covered by our work, gold islands are formed. At this stage, the cathodic current is related to the surface electrochemical reaction..$^{8,9}$

$$
\begin{aligned}
\mathrm{C}(0001)+\mathrm{m} \mathrm{AuCl}_{4}{ }^{-}+ & 3 \mathrm{~m} \mathrm{e}^{-}= \\
& \mathrm{C}(0001)\{\mathrm{Au}\}_{\mathrm{m}}+4 \mathrm{~m} \mathrm{Cl}^{-}
\end{aligned}
$$

where $\{\mathrm{Au}\}_{\mathrm{m}}$ stands for a Au growing cluster consisting of $m$ atoms on the $C(0001)$ surface. Later, clusters further grow into islands by incorporating gold atoms discharged either on the substrate or directly on gold island surfaces,

$$
\begin{aligned}
\mathrm{C}(0001)\{\mathrm{Au}\}_{\mathrm{m}}+\mathrm{AuCl}_{4}{ }^{-}+3 \mathrm{e}^{-}= \\
\mathrm{C}(0001)\{\mathrm{Au}\}_{\mathrm{m}+1}+4 \mathrm{Cl}^{-}
\end{aligned}
$$

Accordingly, the growth of gold islands involves the 

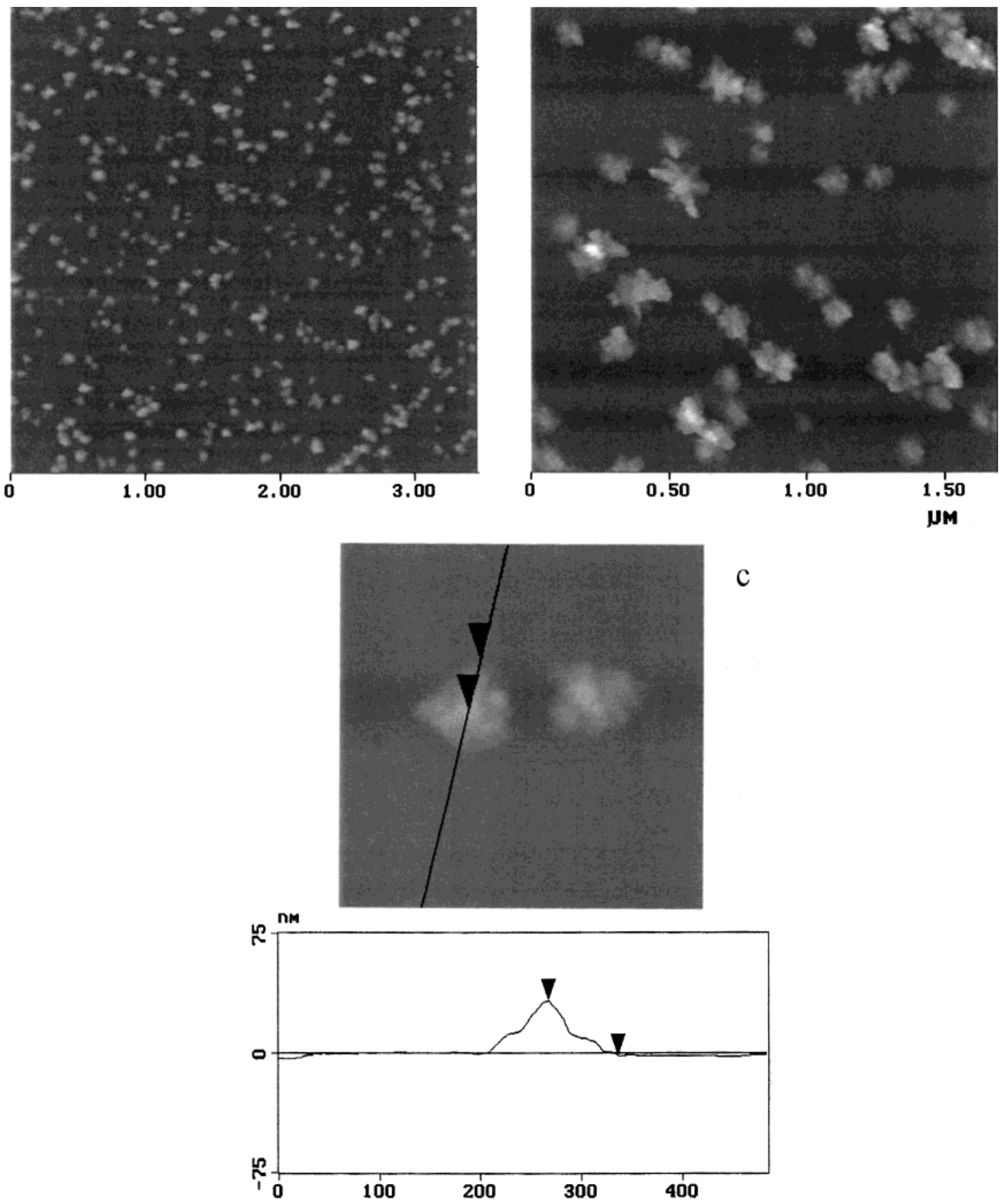

Figure 4. Ex situ STM images (topographic mode) of gold islands on $\mathrm{C}(0001)$ produced from aqueous $5 \times 10^{-4} \mathrm{M} \mathrm{AuCl}_{3} \cdot \mathrm{HCl}+$ $0.5 \mathrm{M} \mathrm{NaClO}_{4}+0.01 \mathrm{M} \mathrm{HClO}_{4}+0.1 \mathrm{mM}$ citric acid under constant flux. (a) $3.5 \times 3.5 \mu \mathrm{m}^{2}$ image, $q=6 \mathrm{mC}^{2} \mathrm{~cm}^{2} ;(\mathrm{b}) 1.7 \times 1.7 \mu \mathrm{m}{ }^{2}$, $q=10 \mathrm{mC} / \mathrm{cm}^{2}$. (c) Cross section of a typical island grown for $q=10 \mathrm{mC} / \mathrm{cm}^{2}$.

transport of $\mathrm{AuCl}_{4}{ }^{-}$ions from the solution to the $\mathrm{C}(0001)$ surface, with its rate being determined by the value of $j_{\mathrm{L}}$ their discharge at defective sites ${ }^{8,9}$ to form nuclei (reaction 4a), followed by their growth producing islands (reaction $4 \mathrm{~b})$.

From the standpoint of electrochemical kinetics, a heterogeneous solid surface consisting of $\mathrm{C}(0001)$ and gold domains is involved in reactions $4 \mathrm{a}$ and $4 \mathrm{~b}$; therefore, the rate of discharge of $\mathrm{AuCl}_{4}{ }^{-}$ions at each domain should be considered. Nevertheless, the gold/C(0001) surface domain ratio increases as the value of $q$ is increased until the overlapping of islands takes place.

The previous work of the authors established that the discharge of $\mathrm{AuCl}_{4}{ }^{-}$ions on $\mathrm{C}(0001)$ is less efficient than that on the gold surface itself. ${ }^{15}$ Then, for the range $6 \leq$ $q \leq 10 \mathrm{mC} / \mathrm{cm}^{2}$, gold island growth should occur mainly by $\mathrm{AuCl}_{4}{ }^{-}$ion discharge at gold domains yielding gold atoms that subsequently shift toward equilibrium locations in the gold lattice. This complex surface relaxation process occurs mainly as a descendent flux of gold atoms over gold domains. The relative contribution and the specific properties of the discharge and relaxation processes determine the shape of gold islands and, accordingly, the value of $f_{i}(i=\mathrm{I}, \mathrm{II}, \mathrm{III})$.

The atom surface diffusion rate on a crystalline solid surface involves activation energy barriers, with their height depending on whether the displacement takes place on a terrace, a step edge, a corner, or any other type of surface defect. Thus, when activation energy barriers at

(15) Blum, B. B.; Salvarezza, R. C.; Arvia, A. J. J. Vac. Sci. Technol. $B, 1999,17,2431$. 


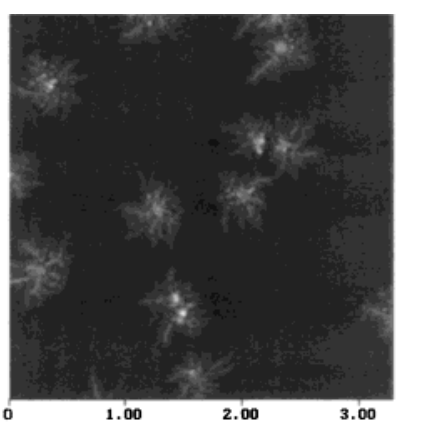

a

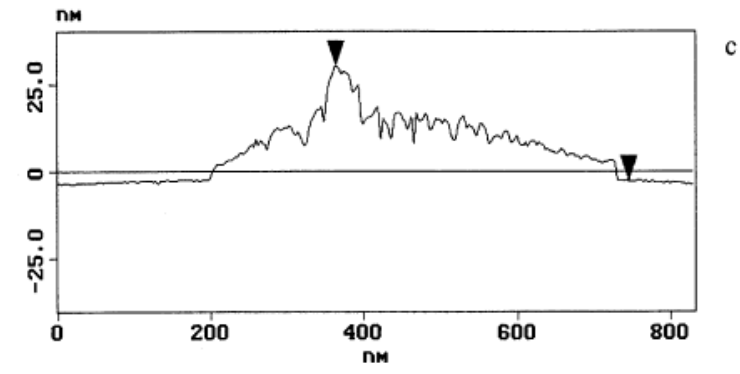

Figure 5. Ex situ STM images (topographic mode) of gold islands on $\mathrm{C}(0001)$ produced from aqueous $5 \times 10^{-4} \mathrm{M} \mathrm{AuCl}_{3}$. $\mathrm{HCl}+0.5 \mathrm{M} \mathrm{NaClO}_{4}+0.01 \mathrm{M} \mathrm{HClO}_{4}+0.92 \times 10^{-2} \mathrm{M} \mathrm{NaCl}$ under constant flux. (a) $3.25 \times 3.25 \mu \mathrm{m}^{2}$ image, $q=6 \mathrm{mC} / \mathrm{cm}^{2}$; (b) $0.7 \times 0.7 \mu \mathrm{m}^{2}, q=6 \mathrm{mC} / \mathrm{cm}^{2}$. (c) Cross section of a typical island resulting from image (b).

step edges are absent, perfect rounded 2D islands growing in the direction parallel to the $\mathrm{C}(0001)$ plane should be produced.

Dendritic gold islands formed on $\mathrm{C}(0001)$ have been characterized as $\mathrm{Au}(111)$ single crystals. ${ }^{8}$ For such a perfect hexagonal surface lattice, the rate of interterrace surface atom diffusion depends on the type of one atom high step edge separating terraces. In this case, two alternate sets of steps (steps B in the (111) direction and steps A in the (100) direction) have been distinguished. ${ }^{4}$ The difference in height of the activation energy barriers for surface diffusion at step edges turns the interterrace, edge, and corner surface atom diffusion anisotropic. Consequently, island growth proceeds preferentially in a trigonal direction. This model for surface atom diffusion has been used to explain $\mathrm{Pt}(111)$ and $\mathrm{Ag}(111)$ island patterns on $\operatorname{Pt}(111)$ resulting from vapor deposition. ${ }^{4}$ The actual situation, however, found for gold island electrodeposition on $\mathrm{C}(0001)$ becomes more complex because these branched islands start from a group of small 3D single-crystal cores with different orientations.

The diffusion length (I) of the gold atoms can be estimated from the ratio $l=D_{\mathrm{s}} / V$, where $D_{\mathrm{s}}$ is the gold surface atom diffusion coefficient and $v$ is island growth velocity in the direction normal to the substrate surface. Gold electrodeposits produced from Solution I always involve to some extent the participation of chloride ions that are present in this solution either from complex goldchloride ion ionic equilibrium ${ }^{16}$ or resulting from interfacial reactions $4 \mathrm{a}$ and $4 \mathrm{~b}$. This means that in any case, either above or below the value of $E_{\mathrm{pzc}}$ of gold in the solution, there is a finite coverage by chloride ions. ${ }^{17}$ Accordingly, taking $D_{\mathrm{s}} \cong 10^{-12} \mathrm{~cm}^{2} / \mathrm{s}^{17}$ for gold atoms in chloride ioncontaining solution and $V \cong 10^{-8} \mathrm{~cm} / \mathrm{s}$ as derived from the

(16) Sillén, L. G. Stability Constant of Metal-Ion Complexes. Section 1: Inorganic Ligands; The Chemical Society, Burlington House: London, $1964 ;$ p 288.

(17) García, P.; Gómez, M.; Salvarezza, R. C.; Arvia, A. J. J. Electroanal. Chem. 1993, 347, 237. value of $j_{\mathrm{L}}$ results in $l=10^{-4} \mathrm{~cm}$, a figure that exceeds the average size of terraces produced in the range of $q$ covered by our work. Therefore, in principle, impinging atoms are able to reach step edges by random walk without nucleus formation on terraces. On the other hand, considering the average terrace size in the range 25-30 $\mathrm{nm}$ for $q=6$ $\mathrm{mC} / \mathrm{cm}^{2}$, and $D_{\mathrm{s}} \cong 10^{-12} \mathrm{~cm}^{2} / \mathrm{s}$, the average residence time of gold atoms at terraces is $\tau \cong 10 \mathrm{~s}$. This figure plays an important role in determining the aspect ratio of island cross sections, as discussed further on.

To understand the nature of surface diffusion processes that take place in the formation of $\mathrm{Au}$ islands, 2D Monte Carlo simulations were performed using the rules of the DDA model, including surface atom diffusion anisotropy for interterrace and corner surface diffusion. ${ }^{4}$ The substrate was simulated by a matrix formed by $256 \times 256$ sites arranged in a (111) lattice. The simulation started from a seed particle placed at the center of the lattice. Then, from a substrate site selected at random, a depositing particle was allowed to move on the surface by random walk to reach the seed for aggregation. Following this rule, a small island was formed. Then, as the island was growing, there was a finite probability that from an island site selected at random a depositing particle moves on the surface island. In this case, the deposited particle also moved on the island by random walk to reach the island edge where it was attached. The rules for particle aggregation at island edges were the following. $P_{\mathrm{A}}$ is the aggregation probability for sites at steps $\mathrm{A} ; P_{\mathrm{B}}$ is the aggregation probability at sites of step $\mathrm{B}$; and $P_{\mathrm{C}}$ is the aggregation probability at corner sites. When $P_{\mathrm{B}}=1>P_{\mathrm{A}}$ $=P_{\mathrm{C}}=0.1$, the snapshot displays dendritic islands with branches emerging at $120^{\circ}$ (Figure 8). This pattern resembles to some extent those observed by STM imaging, although their aspect ratio $f \Longrightarrow 0$ contrasts with $f_{\mathrm{I}}=0.30$ \pm 0.05 resulting from STM cross section analysis of gold islands. Moreover, the $60^{\circ}$ angle formed by the secondary branching direction in gold island growth patterns (Figure 9 ) is an indication that a finite height of the activation energy barrier for surface interterrace diffusion across B-type step edges should be considered. The difference in the values of $f$ and $f_{\mathrm{I}}$ can be attributed to the different finite heights in the activation energy barriers for gold atom surface diffusion across both the A- and B-type step edges. Another possible explanation for the deviation of gold island growth from a perfect $2 \mathrm{D}$ island growth could be given in terms of strains produced by the initial misfit of deposited gold on C(0001). ${ }^{15}$ This, however, does not provide a convincing explanation of the influence of citric acid and chloride ion adsorption on the gold island growth mode.

4.2. The Influence of Citric Acid on Gold Island Growth. Ex situ STM imaging of gold islands produced from citric acid-containing plating solution shows only poor branching and $3 \mathrm{D}$ growth leading to $f_{\mathrm{II}}=0.66 \pm 0.05$. Furthermore, in situ STM images for gold island decay on $\mathrm{Au}(111)$ (Figure 7) demonstrates that citric acid at low concentrations adsorbs preferentially at island edges. It appears that the presence of molecular adsorption of citric acid increases the energy barriers for interterrace and corner gold atom surface diffusion so that, in this case, the distinction between different step edges smears out. Accordingly, the corresponding energy barriers at step edges should be replaced by a new energy barrier, with its height being determined by citric acid molecular adsorption there. The surface diffusion process implies a gradual decrease in gold atom coordination number in going from the upper terrace downward to the top of the reaction coordinate,${ }^{13}$ and its subsequent gradual increase 
$\mathbf{0}$
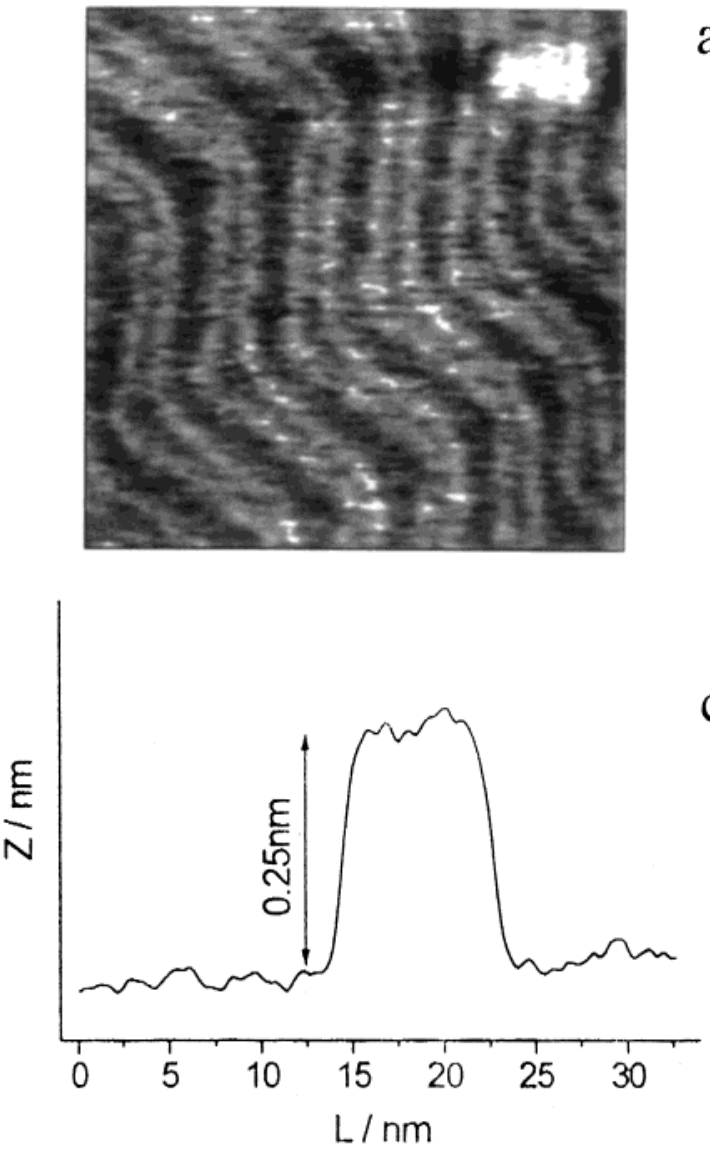

1 min

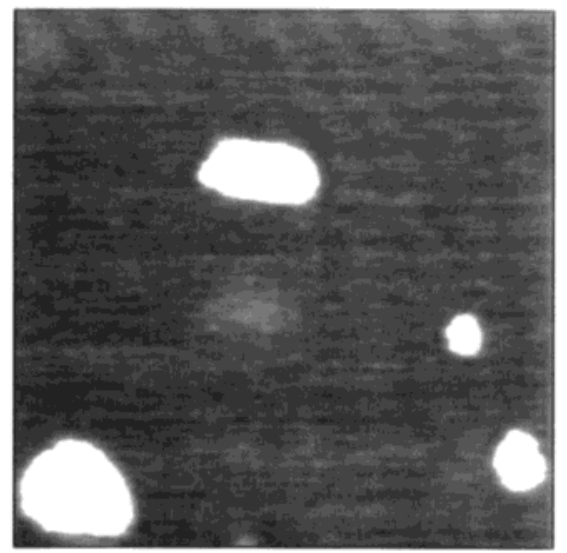

$30 \mathrm{~min}$

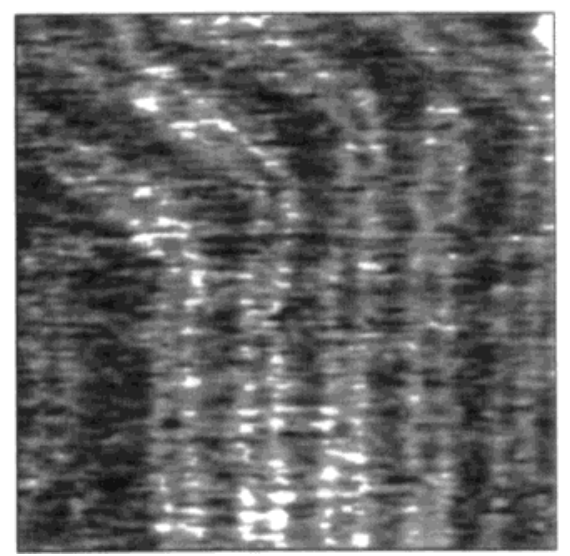

Figure 6. Sequential in situ STM images $\left(38 \times 38 \mathrm{~nm}^{2}\right)(\mathrm{a}, \mathrm{b}, \mathrm{d})$ of a $\mathrm{Au}(111)$ surface domain to follow the $(1 \times 1) \mathrm{Au}(111) \leftrightarrow \mathrm{Au}$ $(22 \times \sqrt{3})$ surface reconstruction and the formation and disappearance of gold islands in aqueous $0.5 \mathrm{M} \mathrm{NaClO}_{4}+0.01 \mathrm{M} \mathrm{HClO}_{4}$. (a) $E=0.20 \mathrm{~V}, t=0$. (b) $E=0.35 \mathrm{~V}, t=1 \mathrm{~min}$. (d) $E=0.20 \mathrm{~V}, t=30 \mathrm{~min}$. The potential of zero charge for Au(111) is $E_{\mathrm{pzc}}=0.25$ $\mathrm{V}$ at $298 \mathrm{~K}$. (c) Cross section of a typical one gold atom high island.

in moving downward to a stable location at the lower terrace. These changes in the coordination number involve first lateral bond breaking and later bond reforming. Molecular adsorption of citric acid at step edges does interfere with the gold surface atom descending flux rate by increasing the corresponding activation energy barrier; that is, it tends to equalize the height of surface diffusion activation energy barriers at different types of step edges assisting a more isotropic island growth. As should then be expected, a higher surface atom diffusion activation energy should imply a lower value of $D_{s}$, as has been found for the surface diffusion of gold atoms on gold in the presence of pyridine..$^{18}$

4.3. The Influence of Sodium Chloride on Gold Island Growth. The reverse situation is observed in gold island formation from a plating solution containing an excess of sodium chloride, a typical Au-Au bond breaking promoter. ${ }^{19}$ In this case, the development of islands with dense radial branching is favored. In contrast to citric acid, the presence of adsorbed chloride ions at step edges assisting gold-chloride complex ion formation would decrease the height of activation energy barriers for gold atom surface diffusion at different step edges favoring 2D growth patterns, as concluded from $f_{\mathrm{III}}=0.14 \pm 0.03$.

4.4. The Aspect Ratio and Surface Diffusion Activation Energy. The aspect ratio derived from the

(18) Alonso, C.; Salvarezza, R. C.; Vara, J. M.; Arvia, Baró, A. M. Electrochim. Acta 1990, 35, 1331.

(19) Trevor, D. J.; Chidsey, C. E. D.; Loiacono, D. N. Phys. Rev. Lett. 1989, 62, 929. cross section analysis of growth patterns appears to be independent of the value of $q$, at least in the range of $q$ covered by our work. Then, constant values of $f_{\mathrm{I}}, f_{\mathrm{II}}$, and $f_{\mathrm{III}}$ indicate in all cases that the growth rate in the vertical and longitudinal direction remains constant. For a perfect hexagonal lattice surface atom diffusion at terraces, step edges and corners participate in surface relaxation in the longitudinal direction. ${ }^{4}$

To deal with this problem, data from different sources should be considered. First, it has been established that the value of $D_{s}$ for gold surface atoms on gold decreases with the adsorption of the organic molecule ${ }^{18}$ and increases with the adsorption of metal complex forming ions, as in the case of chloride ions for gold. ${ }^{19}$ Second, calculations for dendritic growth under diffusion limited metal aggregation for Au(111) and $\mathrm{Ag}(111)$ vapor phase homoepitaxy, ${ }^{4,20}$ which have been made with the effective medium theory (EMT) to get insight into the relaxation processes at island perimeters, provide information about the height of activation energy barriers for surface diffusion on terraces, different types of step edges, and corners.

Let us consider the expression for $D_{s}$ in terms of $\Delta E_{s}{ }^{*}$, the activation energy for gold atom surface diffusion, ${ }^{21}$

$$
D_{s}=D_{o} \exp \left(-\Delta E_{s}{ }^{*} / R \mathrm{~T}\right)
$$

From eq 5 , considering $\Delta E_{S I}{ }^{*} \cong 11 \mathrm{kcal} / \mathrm{mol}^{9}$ derived from

(20) Brune H.; Roeder, H.; Boragno, C.; Kern, K. Phys. Rev. Lett. 1994, 73, 1955. 


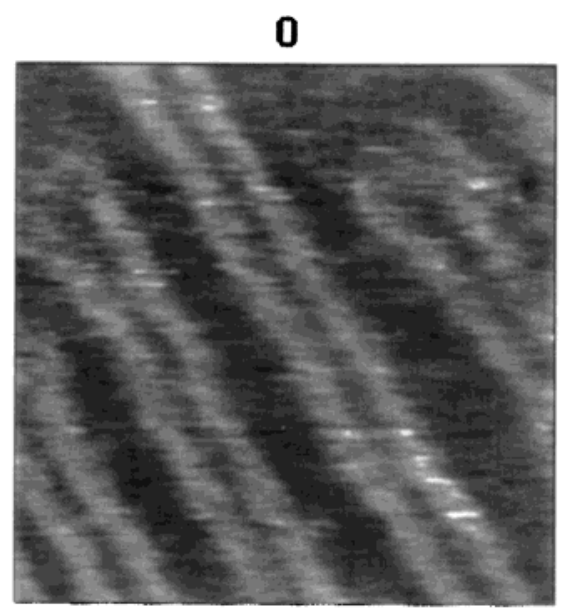

a

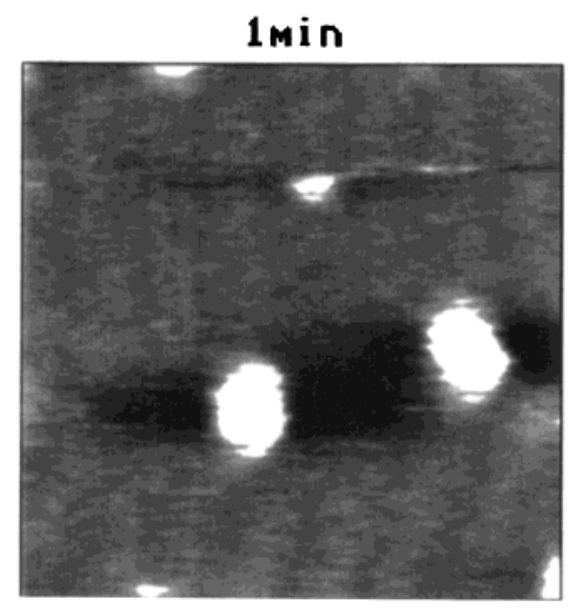

b

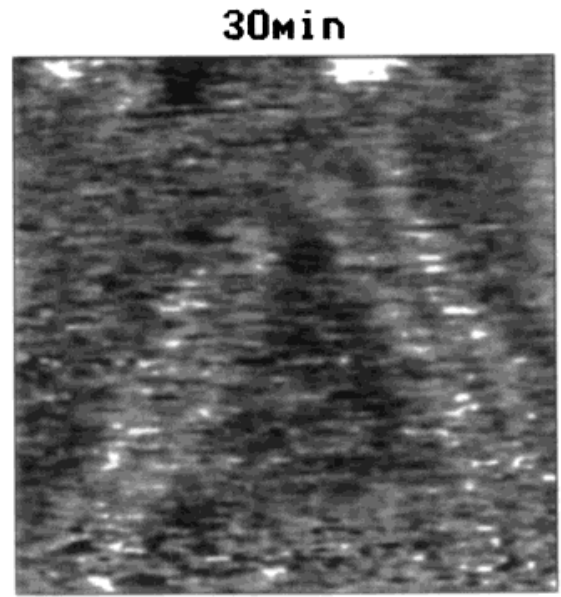

C

Figure 7. Sequential in situ STM images $(25 \times 25) \mathrm{nm}^{2}(\mathrm{a}, \mathrm{b}$, c) of a $\mathrm{Au}(111)$ surface domain to follow the $(1 \times 1) \mathrm{Au}(111) \leftrightarrow$ $\mathrm{Au}(22 \times \sqrt{3})$ surface reconstruction and the formation and disappearance of gold clusters in aqueous $0.5 \mathrm{M} \mathrm{NaClO}_{4}+0.01$ $\mathrm{M} \mathrm{HClO}_{4}+0.1 \mathrm{mM}$ citric acid. (a) $E=0.20 \mathrm{~V}, t=0$. (b) $E=$ $0.35 \mathrm{~V}, t=1 \mathrm{~min}$. (c) $E=0.20 \mathrm{~V}, t=30 \mathrm{~min}$.

data of gold island produced from Solution I, it results in $D_{o} \approx 10^{-4} \mathrm{~cm}^{2} / \mathrm{s}$. This figure lies, within a few orders of magnitude, in the range of those reported for diffusion on

(21) Glasstone, S.; Laidler, K. J.; Eyring, H. In The Theory of Rate Processes; McGraw-Hill: New York and London, 1941, Chapter 9, p 480; Arvia, A. J.; Marchiano, S. L. Los Fenómenos de Transporte en Electroquímica; Comisión de Investigaciones Científicas: Provincia de Buenos Aires, 1971; Chapter 12, p 251.

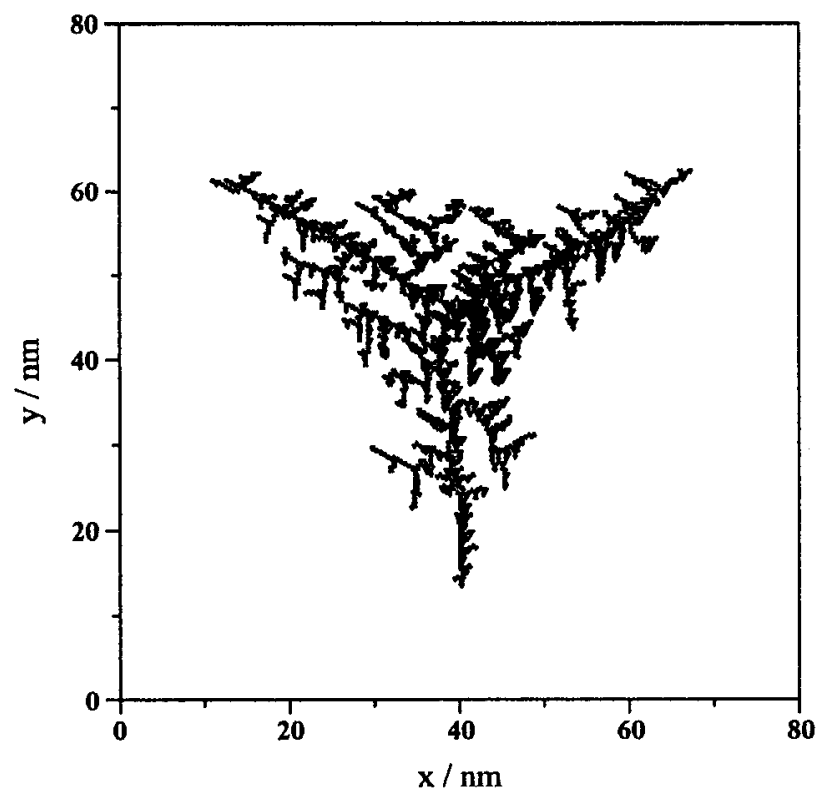

Figure 8. Typical island pattern resulting from Monte Carlo simulation using the growth rules described in the text.

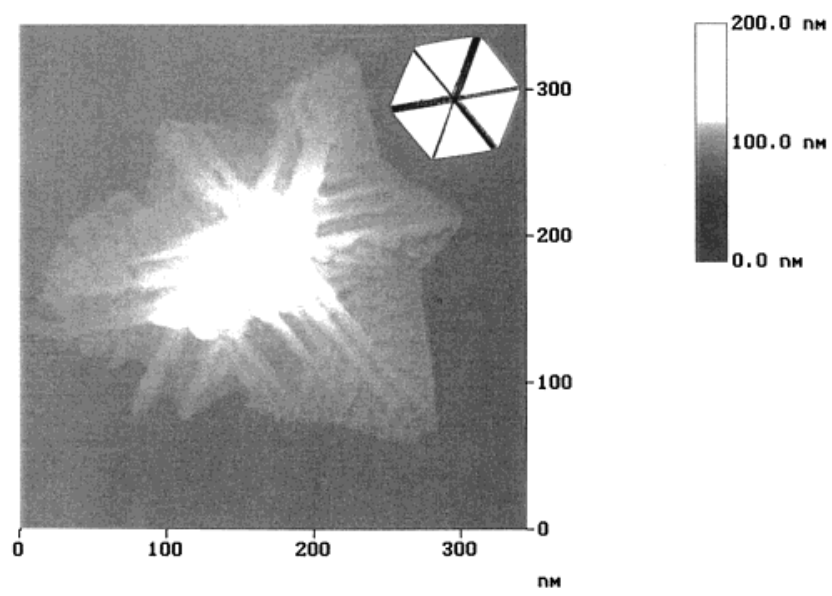

Figure 9. A STM image of a typical gold island produced from Solution I for $q=6 \mathrm{mC} / \mathrm{cm}^{2}$. The main branching directions are indicated by the arrows according to STM image.

solid metal surfaces far below the melting point. ${ }^{22,23} \mathrm{~A}$ cross section analysis of gold island branches formed from Solution I yields $f_{\mathrm{I}}=0.30 \pm 0.05$, a figure that is consistent with ramified $3 \mathrm{D}$ patterns. Accordingly, the value of $f_{\mathrm{I}}$ would represent the difference in the activation energy for anisotropic surface diffusion caused by the existence of A- and B-type step edges; that is, a high activation energy for surface diffusion involving step edges A favoring 3D island growth, and the reverse effect at step edges B favoring the development of radial branching. The difference in the activation energy of the two processes can be estimated from the following relationship

$$
f_{\mathrm{I}} \cong 0.30 \cong \exp \left(\Delta\left(\Delta E_{s}^{*}\right) / R \mathrm{~T}\right.
$$

that leads to a difference in the activation energy for surface atom diffusion to assist $3 \mathrm{D}$ and radial branching growth $\left[\Delta\left(\Delta E_{s}^{*}\right) \cong 0.69 \mathrm{kcal} / \mathrm{mol}\right]$. This figure is consistent with the difference in the activation energy for atom surface diffusion at corners for $\mathrm{A}$ and $\mathrm{B}$ edges $\left(\Delta E_{S A e c}{ }^{*}-\right.$

(22) Gomer, R. Rep. Prog. Phys. 1990, 53, 917; Suni, I. I., Seebauer, E. G. Surf. Sci. Lett. 1994, 301, L235.

(23) Kyuno, K., Ehrlich, G. Surf. Sci. 1999, 437, 29. 
$\Delta E_{s B e c} *=1.61 \mathrm{kcal} / \mathrm{mol}$ ) resulting from calculations using the effective medium theory. ${ }^{4,20}$ Despite limitations imposed by the fact that we are using data calculated for metal/vapor interfaces for our electrochemical interfaces, these figures point out that in our case island branching is also largely determined by corner diffusion at step edges, as has been recently reported. ${ }^{4}$

The analysis of the gold island cross section produced in Solution II yields $f_{\mathrm{II}}=0.66 \pm 0.05$. It is known that the presence of adsorbed molecules decreases the value of $D_{s}$ .${ }^{18}$ This is true for gold islands produced from Solution II, but taking into account reactions $1-3$ the competitive adsorption of chloride ions and citric acid molecules at the reaction interface should be considered. In this case, the increase in height of the activation energy barrier for gold atom surface diffusion produced by the presence of citric acid can be estimated from the $f_{\mathrm{I}} / f_{\mathrm{II}}$ ratio that would represent the surface relaxation rate ratio for gold atom surface diffusion in Solutions I and II. Then, under constant flux and $D_{o}$, the difference in the activation energy for gold atom surface diffusion in those solutions would be reflected by the $f_{\mathrm{I}} / f_{\mathrm{II}}$ ratio, (i.e., $0.45 \cong \exp -\left[\Delta E_{\text {SII }}{ }^{*}-\right.$ $\left.\left.\Delta \mathrm{E}_{\mathrm{sI}}{ }^{*}\right] / R T\right)$, yielding $\Delta\left(\Delta E_{s}{ }^{*}\right) \cong 0.5 \mathrm{kcal} / \mathrm{mol}$. This figure means a decrease in the value of $D_{s}$ due to the presence of citric acid in Solution II, and it agrees qualitatively with the difference in activation energies for either gold or silver island formation calculated from the effective medium theory considering the distinction of A- and B-type step edges. ${ }^{4,20}$ Unfortunately, the errors involved in our measurements prevent an accurate determination of kinetic parameters related to these surface processes.

Finally, the opposite situation is found when an excess of chloride ions are present in the plating solution. The decrease in height of the activation energy barrier for gold atom surface diffusion due to the presence and excess of chloride ions (Solution III) resulting from the $f_{\mathrm{I}} / f_{\mathrm{III}}$ ratio is $\Delta\left(\Delta E_{s}{ }^{*}\right) \cong-0.60 \mathrm{kcal} / \mathrm{mol}$. In this case, $f_{\mathrm{III}}=0.14 \pm 0.03$ indicates the trend to flat gold island formation. In principle, this value of $f$ suggests that low energy barriers for interterrace surface atom diffusion favor radial growth irrespective of the type of step edge. Furthermore, different corner diffusion rates of gold atoms for A- and B-type steps promote dendritic growth. In this case, from the values of $\Delta E_{A c}{ }^{*}=3.36 \mathrm{kcal} / \mathrm{mol}$ and $\Delta E_{B c}{ }^{*}=2.32 \mathrm{kcal} / \mathrm{mol}^{4}$ it follows that

$$
f_{\mathrm{III}} \cong \exp [-(3.36-2.32) / R T]=0.14
$$

This figure agrees with the value of $f_{\text {III }}$ resulting from gold island electrodeposition using Solution III and with earlier conclusions that the island growth direction is largely determined by corner diffusion of gold surface atoms.

According to the analysis just presented, gold island formation can be described as intermediate between two limiting situations; namely, the development of 2D island with $f_{\mathrm{i}} \Longrightarrow 0$ and very low activation energy, and the growth of 3D quasi-symmetric islands with $f_{\mathrm{i}} \Longrightarrow 1$ and relatively high isotropic activation energy for gold atom surface diffusion.

Acknowledgment. This work was financially supported by PIP 4376 and PIP 0897/98 from Consejo Nacional de Investigaciones Científicas y Técnicas, PICT 97-1993 from Agencia Nacional de Promoción Cinetífica y Tecnológica (Argentina), and PI 1999/128 from Gobierno Autónomo Canario (Spain).

LA991130S 